Bull. Austral. Math. Soc.

Vol. 39 (1989) [119-127]

\title{
ON THE TWISTOR SPACE OF THE SIX-SPHERE
}

\author{
Emilio Musso
}

\begin{abstract}
The set of all complex lines of the right-handed Dirac spinor bundle of a standard sixsphere is the total space of the twistor fibration. The twistor space, endowed with its natural Kähler structure, is recognised to be a six-dimensional complex quadric. The relevant group is Spin (7), which acts transitively on the six-quadric, as a group of fiberpreserving isometries. We use a result due to Berard-Bérgery and Matsuzawa to show the existence of a non-Kähler, non sy mmetric, Hermitian-Einstein metric on the six-quadric, which is Spin (7)-invariant.
\end{abstract}

\section{INTRODUCTION}

The present paper was motivated by the following result, which was obtained independently by Berard-Bérgery and Matsuzawa (see [1, 0]): let $F \rightarrow B \rightarrow M$ be a Riemannian submersion with totally geodesic fibres. Assume that the metrics $g_{F}$ $g_{B}$ and $g_{M}$ are Einstein, with Einstein constants $E_{F}, E_{B}$ and $E_{M}$ respectively, and $E_{F}>0$. If $g_{B}$ is not locally a Riemannian product of $g_{F}$ and $g_{M}$, then the metric $g_{B}^{t}$ obtained by scaling the metric on $B$ in the direction of $F$ by a factor $t>0$ is Einstein if and only if $t=1$ or $t=\frac{E_{F}}{E_{M}-E_{F}}$.

Obviously the two metrics above are different if and only if $E_{F} \neq \frac{1}{2} E_{M}$.

Wang and Ziller in [12], pointed out that the only known examples which satisfy the assumptions of the theorem, with $E_{F} \neq \frac{1}{2} E_{M}$, are the Hopf fibrations:

$$
\begin{array}{ll}
S^{3} \rightarrow S^{4 n+3} & \rightarrow H P^{n}, \\
S^{2} \rightarrow \mathrm{CP}^{2 n+1} & \rightarrow \mathrm{HP}^{n}, \\
S^{7} \rightarrow S^{15} & \rightarrow S^{8}
\end{array}
$$

The Riemannian metrics $g_{S^{4 n+3}}^{1}, g_{C P^{2 n+1}}^{1}$ and $g_{S^{15}}^{1}$ are the standard symmetric Einstein metrics. And $g_{S^{4 n+3}}^{t}, g_{C P^{2 n+1}}^{t}, g_{S^{15}}^{t}, t=\frac{E_{F}}{E_{M}-E_{F}}$, are the homogeneous, non symmetric Einstein metrics found by Jensen ([8]), Bourguignon-Karcher ([3]) and Ziller ([14]).

\section{Received 8 April 1988}

The author would like to acknowledge (gratefully) conversations with and inspiration from Franco Tricerri.

Copyright Clearance Centre, Inc. Serial-fee code: 0004-9729/89 \$A2.00+0.00. 
In this paper we will give a new example where the theorem above applies. The standard six-sphere with constant sectional curvature 4 is viewed as a homogeneous space of the group $\operatorname{Spin}(7)$ :

$$
S^{6}=\operatorname{Spin}(7) / S U(4)
$$

The twistor space of $S^{6}$ is realised as the set of all complex lines of the right-handed Dirac spinor bundle. Hence the twistor fibration is given by:

$$
C P^{3} \subset \frac{\operatorname{Spin}(7)}{U(3)} \longrightarrow S^{6}
$$

The normal homogeneous metric $g$ on $\operatorname{Spin}(7) / U(3)$ is a bundle metric, and is Einstein with Einstein constant $E=12$. It is well known that the natural almost complex structure $J$ is integrable, and $(J, g)$ is a Kähler structure. The fibres are totally geodesic complex submanifolds of constant sectional curvature and with Einstein constant $\check{E}=8$.

If we let $\operatorname{Spin}(7)$ act on $\mathrm{P}^{8}$ via its faithful 8 dimensional representation, then it acts transitively on the Grassmannian of the oriented planes of $\mathbf{P}^{8}$ (see [5]), and $U(3)$ is the isotropy subgroup (see [7]). Therefore the twistor space of $S^{6}$ may be recognised to be a six-dimensional quadric of a complex projective space $C P^{7}$ of constant holomorphic sectional curvature 4 .

Since the Einstein constant $E$ of the base is 20 , we see that the Riemannian submersion

$$
\mathrm{CP}^{3} \hookrightarrow Q_{6} \rightarrow S^{6}
$$

satisfies the assumptions of the theorem. Therefore: scaling the metric $g$ on $Q_{6}$ in the direction of $\mathrm{CP}^{3}$ by a factor $t=2 / 3$ we get a Spin (7)-invariant, Hermitian-Einstein metric $g^{\prime}$ on the six-quadric.

We compute the differential of the fundamental two form of $\left(J, g^{\prime}\right)$, and we show that $\left(J, g^{\prime}\right)$ is not Kähler. Since every symmetric Riemannian metric on $Q_{6}$ is a Kähler metric we deduce that $g^{\prime}$ is not symmetric.

\section{Preliminaries}

We let spin (7) be the Lie subalgebra of su(8) whose elements are skew-Hermitian matrices of the form:

$$
S(A, B)=\left|\begin{array}{ll}
A & \bar{B} \\
B & \bar{A}
\end{array}\right|
$$

where: $A \in$ su(4) and $B$ is a $4 \times 4$ complex skew-symmetric matrix satisfying the following conditions:

$$
\bar{B}_{2}^{1}=B_{4}^{3}, \quad \bar{B}_{3}^{1}=-B_{4}^{2}, \quad \bar{B}_{4}^{1}=B_{3}^{2}
$$


The Lie subgroup Spin $(7) \rightarrow \mathrm{SU}(8)$ corresponding to the Lie subalgebra spin (7) is isomorphic (see $[4,5]$ ) to the universal covering group of $\mathrm{SO}(7)$.

The special unitary group $\mathrm{SU}(4)$ is regarded as a subgroup of $\mathrm{Spin}(7)$ by setting:

$$
S \in \mathrm{SU}(4) \rightarrow \mathrm{S}(X, O)=\left\|\begin{array}{ll}
X & 0 \\
O & \bar{X}
\end{array}\right\|
$$

Hence the Lie subalgebra su (4) $\rightarrow$ spin (7) is given by all matrices of the form $\mathrm{S}(A, O)$, $A \in \mathrm{su}(4)$.

We let $m$ be the vector subspace of $\operatorname{spin}(7)$, whose elements are of the form $S(O, B)$, and $B \in O(4, \mathbb{C})$ satisfies (2.1). In the following we will identify $m$ with a six-dimensional Euclidean $A^{6}$ as follows:

$$
\left\|\begin{array}{c}
X^{1} \\
\vdots \\
\vdots \\
X^{6}
\end{array}\right\| \rightarrow\left\|\begin{array}{cccc}
0 & -X^{1}+i X^{2} & -X^{3}+i X^{4} & -X^{5}+i X^{6} \\
X^{1}-i X^{2} & 0 & -X^{5}-i X^{6} & X^{3}+i X^{4} \\
X^{3}-i X^{4} & X^{5}+i X^{6} & 0 & -X^{1}-i X^{2} \\
X^{5}-i X^{6} & -X^{3}-i X^{4} & X^{1}+i X^{2} & 0
\end{array}\right\| .
$$

On $m$ the inner product is obtained by using the Killing form. Then the adjoint representation of Spin( 7 ) restricted to the subgroup SU(4) splits as a direct sum of two irreducible representations. The associated irreducible components of spin (7) are su (4) and $m$; furthermore the representation of SU(4) in $m$ is just the 2:1 spin covering homomorphism of $\mathrm{SU}(4) \simeq \operatorname{Spin}(6)$ onto $\mathrm{SO}(6)$.

Now let us consider $U(3)$ as a subgroup of $\mathrm{SU}(4)$ given by:

$$
Y \in U(3) \longmapsto\left\|\begin{array}{cccc}
Y_{1}^{1} & 0 & Y_{2}^{1} & Y_{3}^{1} \\
0 & (\operatorname{det} Y)^{-1} & & 0 \\
Y_{1}^{2} & 0 & Y_{2}^{2} & Y_{3}^{2} \\
Y_{1}^{3} & 0 & Y_{2}^{3} & Y_{3}^{3}
\end{array}\right\|
$$

We thus have su $(4)=u(3) \oplus n$, where $n$ is identified with $\mathbb{C}^{3}$ by setting:

$$
\left\|\begin{array}{c}
Z^{4} \\
Z^{5} \\
Z^{6}
\end{array}\right\| \mapsto\left\|\begin{array}{cccc}
0 & -\bar{Z}^{4} & 0 & 0 \\
Z^{4} & 0 & Z^{5} & Z^{6} \\
0 & -\bar{Z}^{5} & 0 & 0 \\
0 & -\bar{Z}^{6} & 0 & 0
\end{array}\right\|
$$

It is convenient now to identify $m$ with $C^{3}$ by setting:

$$
\left\|\begin{array}{l}
Z^{1} \\
Z^{2} \\
Z^{3}
\end{array}\right\| \longmapsto\left\|\begin{array}{cccc}
0 & -\bar{Z}^{1} & -Z^{3} & Z^{2} \\
\bar{Z}^{1} & 0 & \bar{Z}^{2} & \bar{Z}^{3} \\
Z^{3} & -\bar{Z}^{2} & 0 & -Z^{1} \\
-Z^{2} & -\bar{Z}^{3} & Z^{1} & 0
\end{array}\right\|
$$


Then the adjoint representation of Spin(7) restricted to the subgroup $U(3)$ decomposes as a direct sum of three irreducible representations, and $u(3) \oplus n \oplus m$ is the associated irreducible decomposition of spin(7). The representation of $U(3)$ in $m$ is given by

$$
Y \in \mathrm{U}(3) \longmapsto \operatorname{det}(Y) Y
$$

and the representation of $U(3)$ in $n$ is:

$$
Y \in \mathrm{U}(3) \longmapsto \operatorname{det}(Y) \bar{Y}
$$

Now let $\omega$ denote the Maurer-Cartan form of $\operatorname{Spin}(7)$; we let $\theta$ be the $\mathrm{R}^{6}$-valued oneform obtained by using the decomposition $\operatorname{spin}(7)=\operatorname{su}(4) \oplus m$ and the identification (2.3). We let $\psi$ be the su (4)-component of $\omega$, and $\phi$ be the $u(3)$-component of $\omega$. We denote by $\sigma^{\prime}$ and $\sigma^{\prime \prime}$ be the $\mathbf{C}^{3}$-valued one-forms given by the $m$ and $n$ components of $\omega$, modulo the identifications (2.6) and (2.5). Then the structure equations of Spin (7) can be written as follows:

$$
\begin{aligned}
& d \sigma^{1}=\left(\phi_{2}^{2}+\phi_{3}^{3}\right) \wedge \sigma^{1}+\bar{\phi}_{1}^{2} \wedge \sigma^{2}+\bar{\phi}_{1}^{3} \wedge \sigma^{3}+\bar{\sigma}^{3} \wedge \sigma^{5}-\bar{\sigma}^{2} \wedge \sigma^{6} \\
& d \sigma^{2}=-\phi_{1}^{2} \wedge \sigma^{1}+\left(\phi_{3}^{3}+\phi_{1}^{1}\right) \wedge \sigma^{2}-\phi_{3}^{2} \wedge \sigma^{3}-\bar{\sigma}^{3} \wedge \sigma^{4}+\bar{\sigma}^{1} \wedge \sigma^{6} \\
& d \sigma^{3}=-\phi_{1}^{3} \wedge \sigma^{1}+\bar{\phi}_{3}^{2} \wedge \sigma^{2}+\left(\phi_{2}^{2}+\phi_{1}^{1}\right) \wedge \sigma^{3}+\bar{\sigma}^{2} \wedge \sigma^{4}-\bar{\sigma}^{1} \wedge \sigma^{5}
\end{aligned}
$$

and

$$
\begin{aligned}
& d \sigma^{4}=\sigma^{3} \wedge \sigma^{2}-\sigma^{2} \wedge \sigma^{3}+\left(\phi_{2}^{2}+\phi_{3}^{3}+2 \phi_{1}^{1}\right) \wedge \sigma^{4}+\phi_{1}^{2} \wedge \sigma^{5}+\phi_{1}^{3} \wedge \sigma^{6}, \\
& d \sigma^{5}=-\sigma^{3} \wedge \sigma^{1}+\sigma^{1} \wedge \sigma^{3}-\phi_{1}^{2} \wedge \sigma^{4}+\left(\phi_{1}^{1}+2 \phi_{2}^{2}+\phi_{3}^{3}\right) \wedge \sigma^{5}-\bar{\phi}_{3}^{2} \wedge \sigma^{5}, \\
& d \sigma^{6}=+\sigma^{2} \wedge \sigma^{1}-\sigma^{1} \wedge \sigma^{2}-\bar{\phi}_{1}^{3} \wedge \sigma^{4}+\phi_{3}^{2} \wedge \sigma^{5}+\left(\phi_{1}^{1}+\phi_{2}^{2}+2 \phi_{3}^{3}\right) \wedge \sigma^{6} .
\end{aligned}
$$

Finally

$$
\begin{aligned}
& d \phi_{1}^{1}+\phi_{2}^{1} \wedge \phi_{1}^{2}+\phi_{3}^{1} \wedge \phi_{1}^{3}=\sigma^{1} \wedge \bar{\sigma}^{1}-\sigma^{2} \wedge \bar{\sigma}^{2}-\sigma^{3} \wedge \bar{\sigma}^{3}-\sigma^{4} \wedge \bar{\sigma}^{4} \\
& d \phi_{2}^{2}+\phi_{1}^{2} \wedge \phi_{2}^{1}+\phi_{3}^{2} \wedge \phi_{2}^{3}=-\sigma^{1} \wedge \bar{\sigma}^{1}+\sigma^{2} \wedge \bar{\sigma}^{2}-\sigma^{3} \wedge \bar{\sigma}^{3}-\sigma^{5} \wedge \bar{\sigma}^{5} \\
& d \phi_{3}^{3}+\phi_{1}^{3} \wedge \phi_{3}^{1}+\phi_{2}^{3} \wedge \phi_{3}^{2}=-\sigma^{1} \wedge \bar{\sigma}^{1}-\sigma^{2} \wedge \bar{\sigma}^{2}+\sigma^{3} \wedge \bar{\sigma}^{3}-\sigma^{6} \wedge \bar{\sigma}^{6} \\
& d \phi_{1}^{2}+\phi_{1}^{2} \wedge \phi_{1}^{1}+\phi_{2}^{2} \wedge \phi_{1}^{2}+\phi_{3}^{2} \wedge \phi_{1}^{3}=-2 \bar{\sigma}^{1} \wedge \sigma^{2}+\bar{\sigma}^{5} \wedge \sigma^{4} \\
& d \phi_{1}^{3}+\phi_{1}^{3} \wedge \phi_{1}^{1}+\phi_{2}^{3} \wedge \phi_{1}^{2}+\phi_{3}^{3} \wedge \phi_{1}^{3}=-2 \bar{\sigma}^{1} \wedge \sigma^{3}+\bar{\sigma}^{6} \wedge \sigma^{4} \\
& d \phi_{2}^{3}+\phi_{1}^{3} \wedge \phi_{2}^{1}+\phi_{2}^{3} \wedge \phi_{2}^{2}+\phi_{3}^{3} \wedge \phi_{2}^{3}=-2 \bar{\sigma}^{2} \wedge \sigma^{3}+\bar{\sigma}^{6} \wedge \sigma^{5}
\end{aligned}
$$

where $\sigma^{\prime}={ }^{T}\left(\sigma^{1} \sigma^{2} \sigma^{3}\right), \sigma^{\prime \prime}={ }^{T}\left(\sigma^{4} \sigma^{5} \sigma^{6}\right)$ and $\phi=\left(\phi_{b}^{a}\right)_{a, b=1,2,3}$ 


\section{THE SPINOR STRUCTURE OF $S^{6}$}

Now let us consider the homogeneous space Spin(7)/SU(4) and the SU(4)principal fibre bundle:

$$
p: \mathrm{SU}(4) \hookrightarrow \operatorname{Spin}(7) \rightarrow \operatorname{Spin}(7) / \mathrm{SU}(4)
$$

Then the $\mathbf{B}^{6}$-valued one form $\theta={ }^{T}\left(\theta^{1} \ldots \theta^{\theta}\right)$ is a tensorial one-form in $\operatorname{Spin}(7)$, which transforms according to the representation $\mathrm{SU}(4)=\operatorname{Spin}(6) \stackrel{\lambda}{\longrightarrow}$ SO $(6)$. Hence the quadratic form $d s^{2}=\sum_{i}\left(\theta^{i}\right)^{2}$ and the exterior form $\theta^{1} \wedge \theta^{2} \wedge \ldots \wedge \theta^{6}$ are projectable on Spin(7)/SU(4), and they define a Riemannian metric $g$ and a volume form.

The su(4)-valued one form $\Psi$ defines a connection form in the bundle (3.1), and the $o(6)$-valued one-form $\lambda(\psi)$ satisfies the following identity:

$$
d \theta=-\lambda(\psi) \wedge \theta .
$$

By using the structure equations (2.10) and (2.11) one has

$$
d \lambda(\psi)_{j}^{i}+\lambda(\psi)_{k}^{i} \wedge \lambda(\psi)_{j}^{k}=4 \theta^{i} \wedge \theta^{j} .
$$

It follows that Spin (7)/SU(4), when endowed with the invariant metric $g$ is a space of constant curvature +4 . We may go further and see that the principal fibre bundle (3.1) is merely the spin double cover of the oriented orthonormal frame bundle of Spin (7)/SU (4) under the metric.

For this reason, we will, from now on, speak interchangably of Spin (7)/ SU (4) and $S^{6}$, even though we have given no explicit isometry between them.

We now consider the right-handed Dirac spinor bundle of $S^{6}$. This is the rank-4 Hermitian complex vector bundle associated with the principal fibre bundle (3.1):

$$
\sum=\operatorname{Spin}(7) X_{\mathrm{SU}(4)} \mathrm{C}^{4} \rightarrow S^{6} .
$$

Since the structure group is SU(4), then the Dirac spinor bundle is naturally equipped together with a complex orientation. Furthermore, the connection $\psi$ induces a Hermitian covariant derivative acting on the cross sections of $\sum$, and it preserves the complex orientation.

\section{ThE TWISTOR FIBRATION OF $S^{6}$}

Let $(M, g, \mathrm{Vol})$ be an even-dimensional oriented Riemannian manifold. Then the set $\mathcal{T}(M)$ of all complex-orthogonal structures on the tangent spaces of $M^{n}$ whose orientation is compatible with the fixed volume form, endowed with the natural topology, is the total space of the twistor bundle $\tau: \mathcal{T}(M) \rightarrow M$. 
Eells and Salamon in $[\boldsymbol{6}]$ show that if $(M, g, \mathrm{Vol})$ is a four-dimensional spin manifold, then the twistor bundle may be viewed as the set of all complex lines of the right-handed Dirac spinor bundle of any spinor structure. In [11] and [13] it is proven that the same construction holds for six-dimensional spin manifolds.

For a $2 n$-dimensional sphere $S^{2 n}$ the twistor bundle $\mathcal{T}\left(S^{2 n}\right)$ is given by

$$
\tau: \frac{\mathrm{SO}(2 n)}{\mathrm{U}(n)} \hookrightarrow \frac{\mathrm{SO}(2 n+1)}{\mathrm{U}(n)} \rightarrow S^{2 n}
$$

Hence $\mathcal{T}\left(S^{2 n}\right)$ is the smooth algebraic variety of all $n$-dimensional totally isotropic linear subspaces of $C^{2 n+1}$.

For $n=6$, using the spinorial approach we get $\mathcal{T}\left(S^{6}\right)=\mathrm{P}\left(\sum\right)$, and hence the twistor fibration is given by

$$
\tau: \mathrm{CP}^{3} \hookrightarrow \frac{\operatorname{Spin}(7)}{\mathrm{U}(3)} \rightarrow S^{6}
$$

It is well-known that $S$ pin (7) acts transitively on the Grassmannian of the oriented planes of $\mathbf{R}^{6}$, via the faithful 8-dimensional representation of Spin (7) in SO (8) (see [5]). The isotropy subgroup is exactly $U(3)$ (see [7]), and hence we may recognise $\mathcal{T}\left(S^{6}\right)$ to be the six-dimensional complex quadric (see [13], where the same result is obtained with different methods).

We now study the geometry of $Q_{\theta}$ determined by the following principal fibre bundle:

$$
\tau: \mathrm{U}(3) \hookrightarrow \operatorname{Spin}(7) \rightarrow Q_{6}
$$

First we notice that $\sigma^{\prime}$ and $\sigma^{\prime \prime}$ are tensorial one forms with respect to (4.3), and they transform according to the representations (2.7) and (2.8). Hence we define an almost Hermitian Spin (7)-invariant structure on $Q_{0}$ by setting

$$
\begin{aligned}
\pi^{*}\left(\Lambda^{(10)} Q_{6}\right) & =\operatorname{Span}\left(\sigma^{1} \ldots \sigma^{6}\right) \\
\pi^{*}\left(g^{\prime}\right) & =\sum_{n=1}^{12}\left(\eta^{a}\right)^{2} \\
\pi^{*}(\Phi) & =-i \sum_{n=1}^{6} \sigma^{i} \wedge \bar{\sigma}^{i}
\end{aligned}
$$

where $\sigma^{1}=\eta^{1}+i \eta^{7}, \sigma^{2}=\eta^{2}+i \eta^{8}, \sigma^{3}=\eta^{3}+i \eta^{5}, \ldots, \sigma^{6}=\eta^{6}+i \eta^{12}$, and $\Phi$ denotes the fundamental two form. 
The structure equations (2.9) and (2.10) imply that the almost-Hermitian structure is a complex Kähler structure.

If we let $\left(\kappa_{a}^{b}\right)_{a, b=1 \ldots 6}$ the restriction of the curvature form of $g^{\prime}$ on $\operatorname{Spin}(7)$, then $-2 i \sum_{a=1}^{6} \kappa_{a}^{a}$ is a projectable two-form. Its projection $\rho$ is the Ricci form, and an easy computations shows that:

$$
-2 i \sum_{a} \kappa_{a}^{a}=-12 i \sum_{a} \sigma^{a} \wedge \bar{\sigma}^{a}=12 \pi^{*}(\Phi)
$$

Therefore $g^{\prime}$ is an Einstein metric with Einstein constant $E^{\prime}=12$. Since $Q_{6}$ is simply connected, then it admits a unique (up to homothety) invariant Kähler-Einstein structure (see $[\mathbf{1 0}])$. Therefore we have that $\left(Q_{6}, g^{\prime}, J\right)$ is the six-dimensional complex quadric of a complex projective space $C P^{\top}$ of constant holomorphic sectional curvature 4.

The bundle $\Lambda^{(10)}\left(Q_{6}\right)$ splits as a direct sum $\mathcal{V} \oplus \mathcal{H}$, where $\mathcal{H}$ is the rank- 3 complex bundle of semi-basic (for $\tau: Q_{6} \rightarrow S^{6}$ ) complex linear $(1,0)$-forms, and $\mathcal{V}=\mathcal{H}^{\perp}$. We notice that

$$
\begin{aligned}
\pi^{*}(\mathcal{H}) & =\operatorname{Span}\left(\sigma^{1} \sigma^{2} \sigma^{3}\right) \\
\pi^{*}(\mathcal{V}) & =\operatorname{Span}\left(\sigma^{4} \sigma^{5} \sigma^{6}\right)
\end{aligned}
$$

Equations (2.10) imply that the subbundle $\mathcal{V} \rightarrow \Lambda^{(10)}\left(Q_{6}\right)$ is a holomorphic, not integrable distribution.

The fibres $C P^{3} \rightarrow Q_{6}$ are the maximal connected integral submanifolds of the exterior differential system

$$
\alpha=0 \quad \forall \alpha \in \mathcal{H}
$$

If we let $j=\mathrm{CP}^{3} \rightarrow Q_{6}$ denote the inclusion, the $j^{*}(\operatorname{Spin}(7)) \rightarrow \mathrm{CP}^{3}$ is a $\mathrm{U}(3)$ principal fibre bundle, and $\sum_{A=4}^{\theta} \sigma^{A} \wedge \bar{\sigma}^{A}$ is a projectable bilinear form representing the induced metric $j^{*}\left(g^{\prime}\right)$. Furthermore, $\sum_{A=4}^{6} \sigma^{A} \wedge \bar{\sigma}^{A}$ gives rise to a well defined complexstructure, and $j^{*}\left(g^{\prime}\right)$ is a Kähler metric.

Notice that $-2 i \sum_{A=4}^{6} \kappa_{A}^{A}$ is a projectible two-form, and its projection is the Ricci form of the fibre.

Since

$$
2 i \sum_{A=1}^{6} \kappa_{A}^{A}=-8 i\left(\sum_{A} \sigma^{A} \wedge \sigma^{A}\right)
$$


we deduce that $j^{*}\left(g^{\prime}\right)$ is an Einstein metric with Einstein constant $\check{E}=8$. Finally we notice that (2.10) implies that the fibres are totally geodesic submanifolds. Since the Einstein constant of $g$ is $E=20$, and obviously the projection $\tau$ is a Riemannian submersion, then we may apply the theorem of Berard Bergery and Matzuzawa and we get the following.

TheOREM. If we scale the standard metric on $Q_{6}$ in the direction of the fibres by a factor $t=\frac{2}{3}$, then we have a Hermitian, Spin (7)-invariant Einstein metric $g^{\prime \prime}$ on $Q_{0}$.

The principal fibre bundle

$$
\pi: \mathrm{U}(3) \rightarrow \operatorname{Spin}(7) \rightarrow Q_{6}
$$

is a reduction of the isotropy bundle $\mathrm{SO}(2) \times \mathrm{SO}(6) \rightarrow \mathrm{SO}(8) \rightarrow Q_{6}$, whose elements are the frames of $S O(8)$ adapted to the twistor projection $\pi: Q_{6} \rightarrow S^{6}$.

Therefore, (4.3) is also a reduction of the unitary frame bundle of the Hermitian manifold $\left(Q_{6}, g^{\prime \prime}, J\right)$. The restriction on $\operatorname{Spin}(7)$ of the canonical one-form of $g^{\prime \prime}$ is the $C^{6}$-valued one form given by:

$$
\left(\sigma^{1}, \sigma^{2}, \sigma^{3}, \sqrt{\frac{2}{3}} \sigma^{4}, \sqrt{\frac{2}{3}} \sigma^{5}, \sqrt{\frac{2}{3}} \sigma^{6}\right) .
$$

Then $-i\left(\sigma^{1} \wedge \bar{\sigma}^{1}+\sigma^{2} \wedge \bar{\sigma}^{2}+\sigma^{3} \wedge \bar{\sigma}^{3}+\frac{2}{3} \sigma^{4} \wedge \bar{\sigma}^{4}+\frac{2}{3} \sigma^{5} \wedge \bar{\sigma}^{5}+\frac{2}{3} \sigma^{6} \wedge \bar{\sigma}^{6}\right)$ is a projectible two-form, representing the fundamental two-form $\Phi^{\prime}$ of $\left(g^{\prime}, J\right)$. Using (2.10) we obtain:

$$
\begin{aligned}
\pi^{*}\left(d \Phi^{\prime}\right)= & \frac{2}{3}\left(\sigma^{2} \wedge \sigma^{3} \wedge \bar{\sigma}^{4}-\sigma^{1} \wedge \sigma^{3} \wedge \bar{\sigma}^{5}\right. \\
& \left.+\sigma^{1} \wedge \sigma^{2} \wedge \bar{\sigma}^{6}-\bar{\sigma}^{2} \wedge \bar{\sigma}^{3} \wedge \sigma^{4}+\bar{\sigma}^{1} \wedge \bar{\sigma}^{3} \wedge \sigma^{5}-\bar{\sigma}^{1} \wedge \bar{\sigma}^{2} \wedge \sigma^{5}\right) .
\end{aligned}
$$

Therefore $d \phi^{\prime} \neq 0$, and hence $g^{\prime \prime}$ is not a Kähler metric. A fortiori $g^{\prime \prime}$ cannot be symmetric or isometric with the standard metric of $Q_{6}$.

\section{REFERENCES}

[1] L. Berard-Bérgery. (unpublished) .

[2] A.L. Besse, Einstein manifolds: Ergeb. Math. Grenzgeb. 3. Folge, Bd. 10 (Springer-Verlag, Berlin, Heidelberg, New York, 1987).

[3] J.P. Bourguignon and H. Karcher, 'Curvature operators: pinching estimates and geometric examples', Ann. Sc. Ecde. Norm. Sup. 11 (1978), 71-92.

[4] R. Bryant, 'Submanifolds and special structures on the octonians', J. Differential Geometry 17 (1982), 185-232. 
[5] R. Bryant, 'Explicit metrics with holonomy $G_{2}$ and Spin (7)'. (I.H.E.S., Bures-sur-Yvette, 1985) (preprint) .

[6] Eells and Salamōn, 'Twistorial construction of harmonic maps of surfaces into four-manifolds', Ann. Scuola Norm. Sup. Pisa Cl. Sci. (4) 12 (1985), 589-640.

[7] A. Gray, 'Vector cross products on manifolds', Thans. Amer. Math. Soc. 141 (1969), $465-504$.

[8] G.R. Jensen, 'Einstein metrics on principal fibre bundles', J. Differential Geom. 8 (1973), 599-614.

[8] T. Matsuzawa, 'Einstein metrics on Fibered Riemannian structures', Kodai Math. J. B (1983), 340-345.

[10] Y. Matsushima, 'Remarks on Kähler-Einstein manifolds', Nagoya Math. J. 46 (1972), $161-173$.

[11] E. Musso, Pseudo-holomorphic curves in the six-sphere (Ph.D Thesis, Washington University, 1987).

[12] M. Wang and W. Ziller, 'On normal homogeneous Einstein manifolds', Ann. Sci. Ecole. Norm. Sup. 18 (1985), 563-633.

[13] P.M. Wong, 'Twistor spaces over 6-dimensional Riemannian manifolds', Mlinois J. Math. 31 (1987), 274-311.

[14] W. Ziller, 'Homogeneous Einstein metrics on spheres and projective spaces', Math. Ann. 250 (1982), 351-358.

Dipartimento di Matematica Pure ed Applicata

Universita Dell-Aquila

via Roma 33

67-100 L'Aquila

Italy 